\title{
A dynamic stochastic model of asset pricing with heterogeneous beliefs
}

\author{
Serena Brianzoni, Roy Cerqueti, Elisabetta Michetti* \\ Dipartimento di Istituzioni Economiche e Finanziarie, \\ Università di Macerata, Italy.
}

\begin{abstract}
???abstract riscritto completamente (eli)??? This paper presents a new stochastic asset pricing model in which, in a context of bounded rationality, beliefs upon future prices are formed via an expectations updating rule characterized by a stochastic multiplicative random variable, that works as an agent-based time dependent weight of the conditional expectation of the fundamental. Basically, we extend the Brock and Hommes (1998) model in a way such that the agent belief upon future prices depends on his confidence on the previsions made by other agents, measured by the proportion of agents belonging to his group and by a confidence parameter. The resulting stochastic dynamical system is firstly analyzed in a deterministic setting, deriving conditions of uniqueness and stability of steady states and proving that with high values of the confidence parameter no complicated dynamics can be exhibited for any values of the other parameters, hence our component has a stabilizing effect on the qualitative dynamics. Differently, for small values of the confidence parameter, we prove the existence of a stability region in the parameters plane where the unique possible dynamics is the convergence to a steady state, while complexity is exhibited outside such region. Starting from the results obtained in the deterministic case, the model is then explored by reintroducing randomness. More precisely, we provide an explicit formula for the probability measure of the stability region and prove that it is robust w.r.t. the confidence parameter (??? giusto ???). In such a way it is possible to know, given the probability distributions of the other random variables, the probability that prices converge to their equlibrium value in the long-run.
\end{abstract}

\footnotetext{
${ }^{*}$ Corresponding author: Elisabetta Michetti, Dipartimento di Istituzioni Economiche e Finanziarie, Università di Macerata, via Crescimbeni 20, 62100 Macerata (Italy). Phone number (+39)0733-2583243, fax number $(+39) 0733-$ 2583205, e-mail: michetti@unimc.it.
} 


\section{Introduction}

???modificato da eli: prima parte uguale, nuovi commenti inseriti???

The traditional approach in economics and finance is based upon a representative agent who is assumed to be rational. This requirement implies that a rational decision maker knows the market equilibrium equations and is able to solve the model. Yet, as has been argued by Simon (1957), knowledge about the economic environment and the model equations is an unrealistic assumption because it is too restrictive. Moreover, in most nonlinear market equilibrium models it is hard to compute the rational expectations equilibrium, even supposing the agent knew all the equilibrium equations.

In recent years, several models in which markets are populated by boundedly rational, heterogeneous agents have been proposed as an alternative to rational expectations (see Conlisk (1996) for a more detailed explanation of bounded rationality). This change involves the following three important aspects. First of all the shift from a representative agent to heterogeneous agent systems. Kirman (2006) argues that heterogeneity plays an important role in economic models and summarizes some of the reasons why the assumption of heterogeneous agents should be considered. One argument is that in a market with homogeneous agents there will be "no trade" because trade will not take place between identical individuals. Another argument considers an evolutionary explanation according to which adaptation and evolution necessarily involve heterogeneity. Second, it is obvious that heterogeneity implies a shift from simple analytically tractable models with a representative, rational agent to a more complicated framework and then a computational approach becomes necessary. Finally, as underlined e.g. in Hommes (2001), in a heterogeneous world, full rationality is impossible since it requires perfect knowledge of the beliefs of other agents and then bounded rationality takes place. The most important feature of this approach is that bounded rationality does not require capability to solve the model and it implies that beliefs and realizations of future variables do not necessarily coincide. More precisely, a boundedly rational agent is modeled as able to form expectations based on an observable information set and he adapts his predictions when some other information becomes available. In order to take decisions, the boundedly rational agent uses simple rules of thumb and adapts his choices when he learns about the economic environment. The resulting Adaptive Learning may or may not converge to a rational expectations equilibrium.

An interesting contribution in the literature evaluating these arguments is given by the theory of evolving selection of predictions, or learning strategies, due to Brock and Hommes (1997) and (1998). The authors propose simple, analytically tractable heterogeneous agent models to show that irrational strategies can survive evolutionary selection. They studied heterogeneity in expectation formation by introducing the concept of Adaptive Belief System to model economic and financial models. More specifically, different types of agents have different beliefs about future variables and prediction selection is based upon a performance measure which is available to all agents. 
Furthermore, the fraction of agents employing each rule is assumed evolving over time instead of being fixed.

In Brock and Hommes (1998) the authors investigate adaptive beliefs in the present discounted value asset pricing model and find complicated dynamics for a large intensity of choice, with an irregular switching between close to fundamental fluctuations and upward or downward price trends. As in many financial models with heterogeneous agents, Brock and Hommes (1998) assume two typical investor types, i.e. fundamentalists and chartists. Fundamentalists believe that the price of an asset is determined by its fundamental value. They sell (buy) assets when their prices are above (below) the fundamental value. In contrast, chartists or technical analysts do not take the fundamental value into account but look for trends in past prices, and prediction is based upon simple trading rules (see Hommes (2006) for an extensive survey of heterogeneous agent models).

Starting from the model studied in Brock and Hommes (1998), we introduce a random variable in the expectations rule. The new ingredient consists of a stochastic term which works as an agentbased time dependent weight of the conditional expectation of the fundamental. The introduction of such a component in the expectation scheme aims at extending the framework of Brock and Hommes (1998) in order to consider that, with bounded rationality, agents try to get informations not only from past observations, but also by taking into account the previsions made by agents belonging to the same group. This mechanism explains how the expectation formation can depend on a sort of confidence on the correctness in the previsions made by other agents, that can be measured by the proportion of agents belonging to the same group, weighted by a confidence parameter. By assuming that such a weight is unitary, independently of time and agent type, we came back to the model of Brock and Hommes (1998).

???verificare come possiamo scrivere il seguente paragrafo o se va tolto??? Furthermore, the model we present takes into account a commonly accepted feature of financial markets: the presence of an imitative behavior among agents. Investors can decide to herd, instead of acting according to the information obtained from market analysis. This phenomenon provides an explanation for the reason for the misalignment between prices and asset values. A recent research field has been developed in order to explain herd behavior among rational agents, through several papers. In Banerjee (1992), Bickhchandani et al. (1992), Chamley and Gale (1996) and Chari and Kehoe (2000), the role of knowledge in markets has been used to study the learning effects of the sequential actions of agents. Avery and Zemsky (1998) proved that it is better to believe in private information than imitate other agents. In our financial market, we model the imitative behavior by assuming that weights depend stochastically on the type-distribution of agents.????

The stochastic dynamical system we obtain is firstly bi-dimensional, but it can be reduced to a one-dimensional one. The state variable is the deviation between theoretical and observed fundamental, and the evolution of the map is studied with respect to the conditional expectation of the 
fundamental $\delta$ (representing what the market expects to gain in the next period from the fundamental), the adjustment term of the proportion of agents $\beta$ (providing information on the movements among the two groups, based on the previous realization of the fundamental) and the confidence parameter $k$. Differently from the results of Brock and Hommes (1998), in our model the presence of the confidence mechanism whose relevance is measured by the confidence adjustment parameter has a stabilizing effect w.r.t. the qualitative asymptotic dynamics. In fact, differently from the results reached on this research line in which this factor has not been taken into account, we prove that if the confidence parameter $k$ is great enough the system is globally stable for any values of the other parameters. Otherwise, for small values of $k$, complex dynamics can be exhibited. In this last case we prove that if $\delta$ and $\beta$ are both small (or large) enough, there exists a stability region in the parameters' plane $(\delta, \beta)$ for which the unique possible dynamics is the convergence to a steady state. The complementary of the stability region is characterized by a large variety of cycles of different orders. Notice that if $\delta$ and $\beta$ are both small (large), then most agents are chartists (fundamentalists), following the new approach, and the forecast on the fundamental provides small (high) values.

After the deterministic study, the stability region is also analyzed by reintroducing randomness. In particular, we provide an explicit formula for its probability measure, starting from a numerical analysis of its boundaries. In such a way it is possible to know the probability that, for any given value of the confidence parameter, the system will converge to a globally stable steady state. Hence given the probability distributions of $\delta$ and $\beta$ and being $k$ exogenous, it is possible to know the probability that the price in the long run will converge to the equlibrium value. Furthermore we prove that our results are robust.

???rivedere con roy il seguente paragrafo??? Then, we propose a bayesian analysis, in order to explore the distribution of $\beta$ and suggest two further research lines: the first one concerns the discovery of an eventual presence of change in persistence of the time-dependent stochastic process describing the adjustment term of the proportion of agents; the second one is related to a possible performing of cluster analyses of the parameter $\beta . ? ? ?$

The remaining part of this paper is organized as follows. In the next section, both the general model and the fundamentalist-chartist new approach model are presented. Section 3 is devoted to the analysis of the deterministic skeleton of the dynamical system. In section 4 some stochastic features of the model are described, in agreement with the deterministic analysis. The final section concludes. 


\section{The asset pricing model}

??? paragrafo riscritto da sere??? The starting point of our model is the framework proposed by Brock and Hommes (1998). The asset pricing model is composed of one risky asset, whose price (ex dividend) per share at time $t$ is $p_{t}$, and one risk free asset which is perfectly elastically supplied at gross return $R=1+r_{f}$. Let $\left\{y_{t}\right\}$ be the stochastic dividend process of the risky asset. The wealth's dynamics $W$ is described by:

$$
W_{t+1}=R W_{t}+\left(p_{t+1}+y_{t+1}-R p_{t}\right) z_{t}
$$

where $z_{t}$ denotes the number of shares of the asset purchased at date $t$.

Let us denote with $E_{t}, V_{t}$ the conditional expectation and conditional variance operators based on a publicly available information set consisting of past prices and dividends. Heterogeneity is introduced via the assumption that agents have different beliefs about the future price of the risky asset. Then, let $E_{h t}, V_{h t}$ be the beliefs of investor type $h$ about the conditional expectation and conditional variance.

The excess return is $p_{t+1}+y_{t+1}-R p_{t}$, and we assume that beliefs about the conditional variance of excess returns are constant and the same for everyone, i.e.

$$
V_{h t}\left(p_{t+1}+y_{t+1}-R p_{t}\right)=\sigma^{2}
$$

for all types $h$. Assume that each investor type is a myopic mean variance maximizer, then for type $h$ the demand for shares $z_{h t}$ solves

$$
\max _{z}\left\{E_{h t}\left(W_{t+1}\right)-\frac{a}{2} V_{h t}\left(W_{t+1}\right)\right\}
$$

i.e.

$$
z_{h t}=\frac{E_{h t}\left(p_{t+1}+y_{t+1}-R p_{t}\right)}{a \sigma^{2}}
$$

where $a$ denotes the risk aversion, which is assumed to be equal for all traders. Let $s_{t}$ denote the supply of shares and $n_{h t}$ the fraction of investors of type $h$ at time $t$. Equilibrium between demand and supply implies

$$
\sum_{h} n_{h t}\left\{\frac{E_{h t}\left(p_{t+1}+y_{t+1}-R p_{t}\right)}{a \sigma^{2}}\right\}=s_{t} .
$$

If there is only one investor type, market equilibrium yields the pricing equation

$$
R p_{t}=E_{h t}\left(p_{t+1}+y_{t+1}\right)-a \sigma^{2} s_{t}
$$

Now consider equation (2) in the special case of zero supply of outside shares, i.e. $s_{t}=0$, for all $t .{ }^{1}$ In other words we analyze a secondary market where we do not have issue of new shares.

\footnotetext{
${ }^{1}$ In making this assumption we follow Brock and Hommes (1998).
} 
Furthermore, in order to get a benchmark notion of the rational expectations fundamental solution $p_{t}^{*}$, consider the information set $\mathcal{F}_{t}$ and the equation

$$
R p_{t}^{*}=E_{t}\left(p_{t+1}^{*}+y_{t+1}\right)
$$

where $E_{t}$ is the conditional expectation on the information set $\mathcal{F}_{t}$.

We define the deviation $x_{t}$ from the benchmark fundamental $p_{t}^{*}$ as

$$
x_{t}=p_{t}-p_{t}^{*} .
$$

Rewrite (1) for the case of zero supply of outside shares to get

$$
R p_{t}=\sum_{h} n_{h t} E_{h t}\left(p_{t+1}+y_{t+1}\right) .
$$

Let us define the class of beliefs we wish to consider.

\subsection{Two Belief types}

In this section we precise the class of beliefs that we wish to introduce, in order to write the final model. Assume:

Assumption 1. All beliefs are of the form

$$
E_{h t}\left(p_{t+1}+y_{t+1}\right)=g_{h t} \cdot E_{t}\left(p_{t+1}^{*}+y_{t+1}\right)+f_{h t}\left(x_{t-1}\right),
$$

where $p_{t}^{*}$ denotes the fundamental, $E_{t}\left(p_{t+1}^{*}+y_{t+1}\right)$ is the conditional expectation of the fundamental on the information set $\mathcal{F}_{t}, x_{t}=p_{t}^{*}-p_{t}$ is the deviation from the fundamental, $f_{h t}$ is a deterministic function and $g_{h t}$ is a stochastic process which can differ across trader types $h$.

As in Brock and Hommes (1998) we assume that the deterministic function $f_{h t}$ is of the simple, linear form:

$$
f_{h t}\left(x_{t-1}\right)=a_{h} x_{t}+b_{h} .
$$

Our work aims at providing an analysis of a market populated by two types of agents. In particular, we assume:

$f_{1 t}\left(x_{t-1}\right)=0$ (i.e. $\left.\left(a_{1}=b_{1}=0\right)\right)$ and $f_{2 t}\left(x_{t-1}\right)=x_{t-1}\left(\right.$ i.e. $\left.\left(a_{2}=1, b_{2}=0\right)\right)$.

According to such deterministic functions type 1 agents believe that the price returns to its fundamental value, whereas type 2 are pure trend chasers. These classes of investors, i.e. fundamentalists and chartists, are included in many interacting agents models in economics and finance. In particular, fundamental traders believe that the price of an asset is determined by its fundamental value, they sell (buy) assets when their prices are above (below) the market fundamental value. In contrast, chartists or technical analysts do not take into account the fundamental value but look for trends in past prices and prediction is based upon simple trading rules. 
We introduce a new ingredient in the expectation schemes defined by Assumption 1 . In fact, differently from Brock and Hommes (1998), equation (6) contains the term $g_{h t}$, that represents a stochastic adjustment factor of the conditional expectation of the fundamental, given the information available at time $t$. Notice that for $g_{h t}=1 \forall h$ and $\forall t$, we find the form of beliefs introduced by Brock and Hommes (1998).

Remembering that in our framework agents are boundedly rational, we assume that they get some information on the behavior of other agents. As a consequence we suppose that $g_{h t}$ depends randomly on the proportion of agents of type $h$ at time $t$ :

$$
g_{h t}=\alpha_{h} n_{h t}+\gamma_{h t},
$$

for any investor type $h$, where $\alpha_{h} \in \mathbb{R}$ and $\gamma_{h t}$ are i.i.d. stochastic processes such that $\gamma_{h t}$ is independent on $\gamma_{k t}$, for any investor type $h \neq k$.

In order to define the beliefs of agents, we have to provide an explicit shape of the adjustment factor $g_{h t}(h=1,2)$ introduced into equation 6 . We observe that the deterministic function $f_{1 t}$ corresponds to fundamentalists, as a consequence we assume that $g_{1 t}$ has to reflect the following conditions:

- the growing confidence in the expectation of the fundamental, as the number of type 1 agents increases;

- the overestimate of the expectation of the fundamental. This fact implies that $g_{1 t}$ is basically greater than 1 .

Hence $g_{1 t}$ can be formalized as follows:

$$
g_{1 t}=k n_{1 t}+\gamma_{t}
$$

where $k \in(0,1)$ and $\gamma_{t}$ i.i.d. such that $E\left[\gamma_{t}\right]=1$.

Notice that the parameter $k$ regulates the dependence of $g_{1 t}$ on the fraction of agents of type 1 at time $t\left(n_{1 t}\right)$. In other words it measures the degree of trust in the expectation made by traders belonging to the same group. As a consequence we can define the parameter $k$ as a confidence parameter.

Contrary to the first group, we assume that the adjustment factor of type 2 agents is negative, with increasing absolute value as the number of agents belonging to group 2 increases. The countertendency with respect to the first group allows us to use the same proportionality parameter $k$, and to define $g_{2 t}$ as

$$
g_{2 t}=-k n_{2 t}
$$


Summarizing, our belief types are driven by two different point of view, which provide new versions of fundamentalist and chartist traders respectively.

In line with the existing literature on heterogeneous agents and, in particular, with the framework of Brock and Hommes, we can specialize the new version of fundamentalist belief, which characterizes the first group of agents, as the most sophisticated predictor.

The introduction of new factors in the expectation schemes aims at extending the framework of Brock and Hommes (1998) and it contributes to the literature on heterogeneous agents. More precisely, the proposed extension on the expectation schemes considers the presence of the term $g_{h t}$ which depends on $n_{h t}$, according to the fact that agents try to get information on the choice of other agents. Moreover proportions of agents vary during time, therefore we have switch phenomenon of the agent forecasts' approach, that implies a change in the adjustment factors $g$ 's as time varies. The size of such inversions provides information on the price dynamics. In other words agents are not purely price takers, but they can modify the path of the price evolution of a risky asset.

In particular, we assume that proportions of agents depend on the known deviation from the fundamental at the previous time:

$$
x_{t-1}=p_{t-1}-p_{t-1}^{\star}
$$

and on the size of the switch between the adjustment factors at the previous time:

$$
m_{t-1}=g_{1, t-1}-g_{2, t-1}
$$

where $g_{1 t}$ and $g_{2 t}$ are defined in (8) and (9). The dependence on the term $x_{t}$ is due to the fact that investors try to get information from the errors made at the previous time, that are measured by the deviation from the fundamental at time $t-1$. To keep the model as general as possible, such dependence is assumed to show randomness. In other words, we suppose that a stochastic process $\beta_{t}$ exists with support in $(0,+\infty)$ such that

$$
n_{c t}=\exp \left\{-\left(\beta_{t} x_{t-1}^{2}+m_{t-1}^{2}\right)\right\}, \quad n_{f t}=1-n_{c t} .
$$

It will be clear from Formula 10 that, as in Brock and Hommes (1998), if $\beta$ is infinite the entire mass of traders uses the most sophisticated strategy. Nevertheless, differently from Brock and Hommes (1998), fractions of agents depend on the deviation from the fundamental and on the size of the switch between the adjustment factors at the previous time. This prediction selection is important. After the deviation from the fundamental and the size of the switch between the adjustment factors at the previous time have been observed, the new fractions are determined. These updating fractions determine the next adjustment factors which interferes in the expectation formation at time $t$. This is in agreement with the fact that bounded rationality does not require capability to solve the model and agents adapt their predictions when some other information becomes available.

Finally, we wish to introduce the following random variable in the expectation schemes:

$$
\delta_{t+1}=E_{t}\left[p_{t+1}^{*}+y_{t+1}\right]
$$


since the forecast at time $t$ of the fundamental value is a stochastic expected value. In fact, differently from Brock and Hommes (1998) we assume a generic stochastic dividend process $y_{t}$ of the risky asset and we consider explicitly the role of the random variable $E_{t}\left[p_{t+1}^{*}+y_{t+1}\right]$ in the expectation formation. ??? VA BENE? POSSIAMO DIRE DI +

Let us go to write the final version of our model. From Assumption 1 and formula (5), we obtain the following equilibrium equation:

$$
R x_{t}=n_{2 t}\left[\left(g_{2 t}-1\right) E_{t}\left[p_{t+1}^{*}+y_{t+1}\right]+f_{2 t}\left(x_{t-1}\right)\right]+n_{1 t}\left[\left(g_{1 t}-1\right) E_{t}\left[p_{t+1}^{*}+y_{t+1}\right]+f_{1 t}\left(x_{t-1}\right)\right],
$$

where $g$ 's and $n$ 's are defined, respectively, in (7) and (10). Recalling that $\delta_{t+1}=E_{t}\left[p_{t+1}^{*}+y_{t+1}\right]$, equation (11) becomes

$$
R x_{t}=n_{2 t}\left[\left(g_{2 t}-1\right) \delta_{t+1}+f_{2 t}\left(x_{t-1}\right)\right]+n_{1 t}\left[\left(g_{1 t}-1\right) \delta_{t+1}+f_{1 t}\left(x_{t-1}\right)\right] .
$$

The bivariate stochastic dynamical system we wish to study is obtained by combining equations (??) and (12). After the opportune substitutions and recalling that $f_{1 t}=0$ and $f_{2 t}\left(x_{t-1}\right)=x_{t-1}$, we get:

$$
T:=\left\{\begin{array}{l}
R x_{t}=e^{-\left(\beta_{t} x_{t-1}^{2}+m_{t-1}^{2}\right)}\left\{\left[-k e^{-\left(\beta_{t} x_{t-1}^{2}+m_{t-1}^{2}\right)}-1\right] \delta_{t+1}+x_{t-1}\right\}+ \\
\left(1-e^{-\left(\beta_{t} x_{t-1}^{2}+m_{t-1}^{2}\right)}\right)\left\{\left[k\left(1-e^{-\left(\beta_{t} x_{t-1}^{2}+m_{t-1}^{2}\right)}\right)+\gamma_{t}-1\right] \delta_{t+1}\right\} \\
m_{t}=k+\gamma_{t}
\end{array}\right.
$$

The following sections contain the analysis of the dynamical system formalized in (13). The study will be conducted in two steps. First of all, in section 3, we analyze the deterministic skeleton of the map, by reproducing the parameters of the system in a deterministic framework. Then, in section 4 we return to the stochastic setting by exploring the randomness of the system, maintaining the analysis in line with the deterministic findings. To reach this goal, the asymptotic distributions of the stochastic parameters are derived in agreement with the stability features of the deterministic equilibria.

\section{The deterministic skeleton}

???rivisto da eli e inseriti alcuni commenti??? In order to focus on the deterministic non-linear asset price dynamics, we can state some hypotheses on the stochastic parameters intervening in the model. Since $E\left[\gamma_{t}\right]=1$, for each $t$, we can assume consistently that $\gamma_{t}=1 \forall t$. Moreover, $\delta_{t}$ represents the conditional expectation of the fundamental. From equation (3), it follows that $E_{t}\left[\delta_{t+1}\right]>0$, and as a consequence we can assume that $\delta_{t}$ is a stochastic process with positive support. In the deterministic framework, we define $\delta_{t}=\delta>0$. Finally, we stress that $\beta_{t}$ is a stochastic process with support in $(0,+\infty)$, therefore we assume that $\beta_{t}=\beta>0 \forall t$.

Taking into account the previous assumptions on the parameters, system (13) is given by: 


$$
T:=\left\{\begin{array}{l}
R x_{t}=e^{-\left(\beta x_{t-1}^{2}+m_{t-1}^{2}\right)}\left\{\left[-k \cdot e^{-\left(\beta x_{t-1}^{2}+m_{t-1}^{2}\right)}-1\right] \delta+x_{t-1}\right\}+ \\
+\left(1-e^{-\left(\beta x_{t-1}^{2}+m_{t-1}^{2}\right)}\right)\left[k\left(1-e^{-\left(\beta x_{t-1}^{2}+m_{t-1}^{2}\right)}\right)+\gamma-1\right] \delta \\
m_{t}=k+\gamma
\end{array} .\right.
$$

Remark 2. System (14) reduces to the one-dimensional dynamic system given by:

$$
x_{t}=\phi\left(x_{t-1}\right)=\frac{1}{R}\left[e^{-\left(\beta x_{t-1}^{2}+(k+1)^{2}\right)}\left(x_{t-1}-\delta(1+2 k)\right)+\delta k\right] .
$$

The parameters we want to consider in order to study the dynamic evolution of the map (15) are given by $\beta$ and $\delta$. This choice is driven by the financial meaning of $\delta$ and $\beta$. In fact $\delta$ is the conditional expectation of the fundamental, representing what the market expects to gain in the next period from the fundamental, given the available information set. On the other hand, $\beta$ is the adjustment term for the proportions of agents, and it is related to the deviation between theoretical and observed fundamental. Roughly speaking, the parameter $\beta$ provides information on the movements among fundamentalist and chartist groups, based on the previous realization of the fundamental. More specifically, high values of $\beta$ involve an increasing proportion of fundamentalists. It is clear from (10) that the special limiting case $\beta \rightarrow+\infty$ corresponds to the case where in each period all agents choose the most sophisticated predictor, i.e. fundamentalist beliefs. The joint analysis of the map with respect to $\delta$ and $\beta$ explains the relationship between changes in the proportion of fundamentalist agents and forecasts on the fundamental value.

In the following, we state some general results for the map (15). In particular, we first analyze the number of fixed points owned by the map and their stability and, then, we concentrate our attention on several bifurcations which route to complexity.

Lemma 3. (Existence and stability of steady states)

Let $k \in(0,1)$. Then $\exists k_{1} \in(0,1)$ such that $\forall \delta>0, \beta>0$ :

1. if $k \geq k_{1}$ there are two possibilities:

(a) there is one globally stable steady state $x_{1}^{\star} \geq 0$,

(b) there are three steady states $0 \leq x_{1}^{\star}<x_{2}^{\star} \leq x_{3}^{\star}<\frac{\delta k}{R}$, the steady state $x_{2}^{\star}$ is unstable and $x_{1}^{\star}, x_{3}^{\star}$ are (locally) stable.

In the case $k=k_{1}$, the steady state $x_{1}^{\star}$ is the fundamental $\left(x_{1}^{\star}=0\right)$.

2. if $k<k_{1}$ there are two possibilities:

(a) there is one steady state $x_{1}^{\star}<0$,

(b) there are three steady states $x_{1}^{\star}<0$ and $0<x_{2}^{\star} \leq x_{3}^{\star}<\frac{\delta k}{R}$, the steady state $x_{2}^{\star}$ is unstable and $x_{3}^{\star}$ is (locally) stable. 
(a)

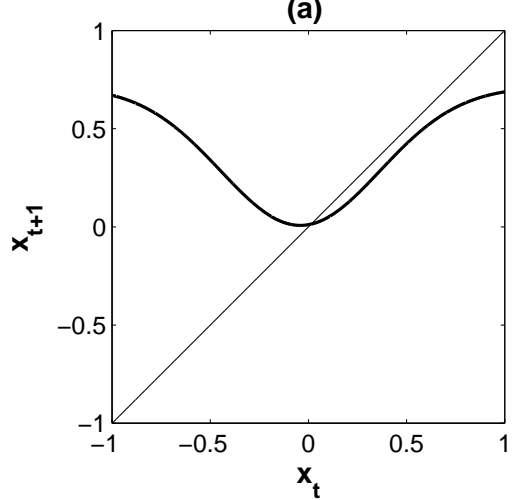

(b)

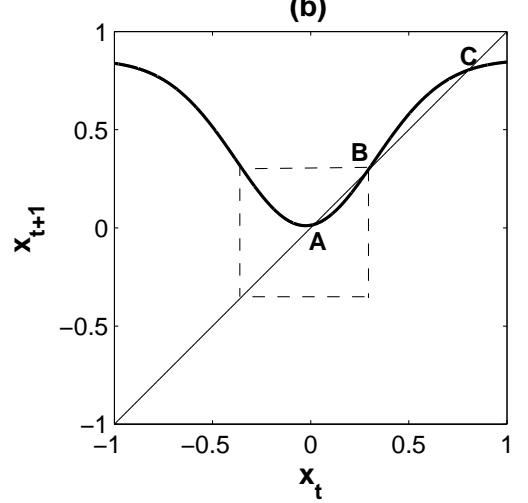

Figure 1: Map (15) for $k=0.3 \geq k_{1}$ and $R=1.05$. (a) For $\beta=3$ and $\delta=2.5$ a unique positive globally stable fixed point exists. (b) For $\beta=4$ and $\delta=3$ there are three positive fixed points: point $B$ is a repelling fixed point, while $A$ and $C$ are locally stable.

Proof. In order to prove the existence of steady states, consider the following equation:

$$
\phi\left(x^{\star}\right)=x^{\star}
$$

where function $\phi$ is defined as in equation (15). From equation (16) we get that a steady state $x^{\star}$ must satisfy $e^{\beta x^{\star 2}+(k+1)^{2}}=\frac{x^{\star}-\delta(1+2 k)}{R x^{\star}-\delta k}$. Define $g(x)=e^{\beta x^{2}+(k+1)^{2}}$ and $h(x)=\frac{x-\delta(1+2 k)}{R x-\delta k} \forall x<\frac{\delta k}{R}$, then straightforward computations yield the following results:

- $x=0$ is the unique critical point of $g$ which is a minimum point,

- $h(x)$ is positive and strictly increasing and such that $\lim _{x \rightarrow-\infty} h(x)=\frac{1}{R}$ while $\lim _{x \rightarrow \frac{\delta k}{R}} h(x)=$ $+\infty$.

Hence, if $g(0) \geq h(0)$ both the following cases are possible: there exists a unique steady state (which is the fundamental $x_{1}^{\star}=0$ in the case $g(0)=h(0)$, while $0<x_{1}^{\star}<\frac{\delta k}{R}$ in the case $g(0)>h(0)$ ) or there are two more steady states $0<x_{2}^{\star} \leq x_{3}^{\star}<\frac{\delta k}{R}$. Similarly, if $g(0)<h(0)$ there are two possibilities: one steady state $x_{1}^{\star}<0$ or three steady states $x_{1}^{\star}<0,0<x_{2}^{\star} \leq x_{3}^{\star}<\frac{\delta k}{R}$. Equation $g(0)=h(0)$ (i.e. $\left.e^{(k+1)^{2}}=\frac{1+2 k}{k}\right)$ has a unique solution $k_{1}$, being functions on the left and on the right side increasing and decreasing w. r. t. $k \in(0,1)$, and $\left(e^{(k+1)^{2}}<\frac{1+2 k}{k}\right)_{\left.\right|_{k \rightarrow 0}},\left(e^{(k+1)^{2}}>\frac{1+2 k}{k}\right)_{\left.\right|_{k=1}}$.

The stability results follow directly from graphical analysis of function $\phi(x)$. More precisely, $\phi(x)$ has one minimum point $x_{m}<0$ and one maximum point $x_{M}>0$. Moreover, $\lim _{x \rightarrow \pm \infty} \phi(x)=\frac{\delta k}{R}$ and $\phi(0) \geq 0$ if and only if $k \geq k_{1}$. In both the cases $k \geq k_{1}$ and $k<k_{1}$, the two positive fixed points $x_{2}^{\star}, x_{3}^{\star}$ are created via fold bifurcation.

As stated in Lemma 3, a $k$-value given by $k_{1} \simeq 0.296588$ does exist such that if $k \geq k_{1}$ only simple dynamics is exhibited by the map (15). In fact $\phi^{\prime}\left(x_{i}^{\star}\right) \geq 0$ for $i=1,2,3$ so that the dynamic 
system is unable to produce cycles or more complex dynamics. The cases $(a)$ and (b) discussed in Lemma 3 point 1 are presented in figure 1. In panel $(a)$ the case with a unique non negative fixed point $x_{1}^{\star}$ is presented which attracts trajectories starting from every i.c. as $0 \leq \phi^{\prime}\left(x_{1}^{\star}\right)<1$. A different situation is shown in panel $(b)$ concerning the case with three non negative fixed points: $A=x_{1}^{\star}, B=x_{2}^{\star}$ and $C=x_{3}^{\star}$ such that $B$ is unstable while the other fixed points are (locally) stable. More precisely, let $\left(x_{2}^{\star}\right)_{-1}$ be the preimage of $x_{2}^{\star}$ different from $x_{2}^{\star}$, i.e. $\phi\left(\left(x_{2}^{\star}\right)_{-1}\right)=x_{2}^{\star}$, then initial conditions belonging to $\left(-\infty,\left(x_{2}^{\star}\right)_{-1}\right) \cup\left(x_{2}^{\star},+\infty\right)$ generate trajectories converging to the steady state $C$, while the set $\left(\left(x_{2}^{\star}\right)_{-1}, x_{2}^{\star}\right)$ is the basin of attraction of the fixed point $A$. Observe that the case with three fixed points can emerge only for $k \in\left[k_{1}, k_{1}+\epsilon\right)$ with $\epsilon$ sufficiently small, as if $k$ is large enough, i.e. $k \rightarrow 1$, our system admits a unique positive globally stable steady state $\forall \beta, \delta$. As a consequence the presence of the confidence parameter $k$ represents a stabilizing factor. In fact our model cannot produce complicated dynamics if $k$ is great enough for any values af the other parameters, differently from Brock and Hommes (1998) where complex dynamics is exhibited for large values of the intensity of choice.

Consider now the case $k<k_{1}$. As stated in Lemma 3 point 2, also in this case two different scenarios may occur, i.e. one or three fixed points may exist. In both cases $x_{1}^{\star}<0$ while the other fixed points $0<x_{2}^{\star} \leq x_{3}^{\star}$, eventually owned by $\phi$, belong to the increasing portion of $\phi$ so that $x_{2}^{\star}$ is unstable while $x_{3}^{\star}$ is locally stable and no more complicated features can be related to the positive steady states.

Let us go to focus on the study of the local dynamics around the fixed point $x_{1}^{\star}$. Let $x_{m}$ denote the minimum point of our map, given by $x_{m}=\frac{1}{2} \delta(1+2 k)-\frac{1}{4 \beta} \sqrt{4 \beta^{2} \delta^{2}(1+2 k)^{2}+8 \beta}$. If $x_{m}>\phi\left(x_{m}\right)$ then $x_{1}^{\star}<x_{m}$ and consequently $\phi^{\prime}\left(x_{1}^{\star}\right)<0$ so that complicated dynamics can be produced via period doubling bifurcations as some parameters are varied. Figure 2 confirms this fact.

In panel (a) the fixed point $x_{1}^{\star}$ is stable being $-1<\phi^{\prime}\left(x_{1}^{\star}\right)<0$; in panel $(b)$, as $\beta$ is increased, the eigenvalue $\phi^{\prime}\left(x_{1}^{\star}\right)$ has crossed -1 and, consequently, the fixed point has lost its stability via period doubling bifurcation: a stable two period cycle has been created. We expect that several period doubling bifurcations are produced if the parameter $\beta$ still increases. The bifurcation diagram in figure 3 confirms this fact and the period doubling route to chaos is exhibited.

Geometrical considerations and numerical simulations enable us to conclude that if $k$ is small enough (i.e. $k<k_{1}$ ) and $x_{1}^{\star}<x_{m}$, the negative fixed point owned by $\phi$ may be stable or unstable. If it is unstable a more complex attractor exists, as cycles of period $m$ or a more complex (even strange) invariant set. We denote with $\mathcal{A}$ the attractor of $\phi$ around $x_{1}^{\star}$. As previously underlined it may consist of the steady state, a period- $m$ cycle or a more complex set.

We now consider the role of the parameters $\delta, \beta$ responsible for complicated dynamics, being $k$ small enough. To reach this goal, it will be useful to consider the limiting cases $(\delta, \beta) \rightarrow(0,0)$ and $\beta \rightarrow+\infty$, important in understanding the cases with small values of the couple $(\delta, \beta)$ and large but 
(a)



(b)

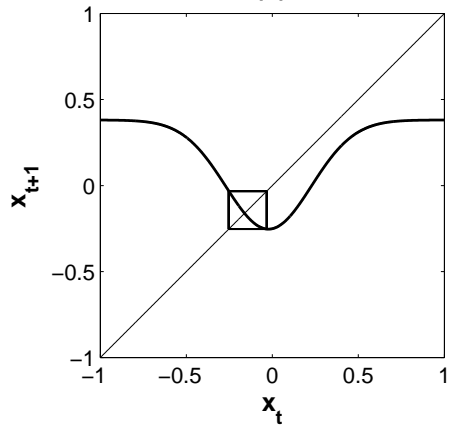

Figure 2: Koneig-Lamerary staircase diagram of map (15) for $k=0.2<k_{1}, R=1.05$ and $\delta=2$ with initial condition $x_{0}=x_{m}$. (a) For $\beta=4, x_{1}^{\star}$ is (locally) stable. (b) For $\beta=8$, a locally stable two period cycle has been created.

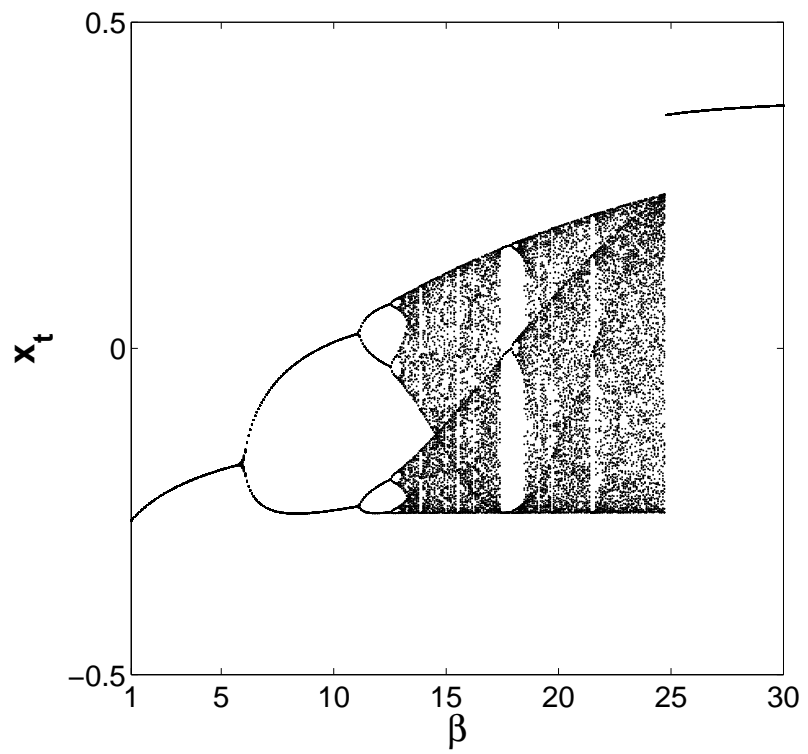

Figure 3: Bifurcation diagram of map (15) with respect to $\beta$ for $k=0.2<k_{1}, R=1.05$ and $\delta=2$.

The bifurcation cascade is presented. 
finite values of the parameter $\beta$. As previously stated, when $\beta$ increases, more and more agents use the most sophisticated predictor, the fundamentalist belief system. In the extreme case $\beta \rightarrow+\infty$ all agents are fundamentalists, in the other extreme case $\beta \rightarrow 0$ no switching at all takes place and our model reduces to one with fixed and $k$-dependent proportions of agents. The next result describes what happens in these limiting cases.

Lemma 4. (The limiting cases) Let $k \in(0,1)$.

1. For $(\delta, \beta) \rightarrow(0,0)$ then $x_{0}=0$ is the unique, globally stable steady state.

2. For $\beta \rightarrow+\infty$ and $\forall \delta>0$, there exists a unique globally stable steady state $x^{\star}=\frac{\delta k}{R}$.

Proof. The results follow immediately from the limiting forms of $\phi(x, \delta, \beta)$ :

$$
\lim _{(\delta, \beta) \rightarrow(0,0)} \phi(x, \delta, \beta)=\frac{1}{R} e^{-(k+1)^{2}} x
$$

and

$$
\lim _{\beta \rightarrow+\infty} \phi(x, \delta, \beta)=\frac{\delta k}{R} .
$$

As a consequence, as long as $\beta$ and $\delta$ are both small enough, the system globally converges to a unique steady state given by the fundamental.

The dynamics is still simple for large but finite values of the parameter $\beta$ as shown by the bifurcation diagram in figure 3 where the fixed point is globally stable $\forall \beta>\bar{\beta}$ being $\bar{\beta} \simeq 25$. This result holds for all $\delta>0$. Notice that $\phi(x, \delta, \beta)$ diverges as $\delta$ goes to $+\infty$, so that we have to concentrate on intermediate values of the couple $(\delta, \beta)$ in order to investigate the cases in which $\mathcal{A}$ consists of more than one point (i.e. cycles or a more complex set). For this reason we focus on the two-dimensional bifurcation diagram as both $\delta$ and $\beta$ vary taking into account small values of $k$.

The following figures 4 and 5 contain cycle cartograms showing a two-parametric bifurcation diagram qualitatively. Each color represents a long-run dynamic behaviour for a given point in the parameter plane $(\delta, \beta)$ and for the initial condition $x_{0}=x_{m}$. Observe a large variety of cycles of different orders. Also note that, as typical in one-dimensional bimodal dynamic maps, several period doubling and period halving cascades exist (see Hommes, 1994). These figures confirm the attained result, i.e. if $(\delta, \beta) \rightarrow(0,0)$ or $\beta \rightarrow+\infty$ the fixed point is globally stable.

As it has been proved for small values of $k$ the system is globally stable if $\beta$ is not too large, as in Brock and Hommes (1998). Anyway a different scenario is presneted as $\beta$ increases. In fact for intermediate values of $\beta$ complicated dynamics can emerge. 
We call stability region the set of points of the parameter plane $(\delta, \beta)$ for which the unique possible dynamics is the convergence to a steady state. In figures 4 and 5 this set is represented by the blue region.

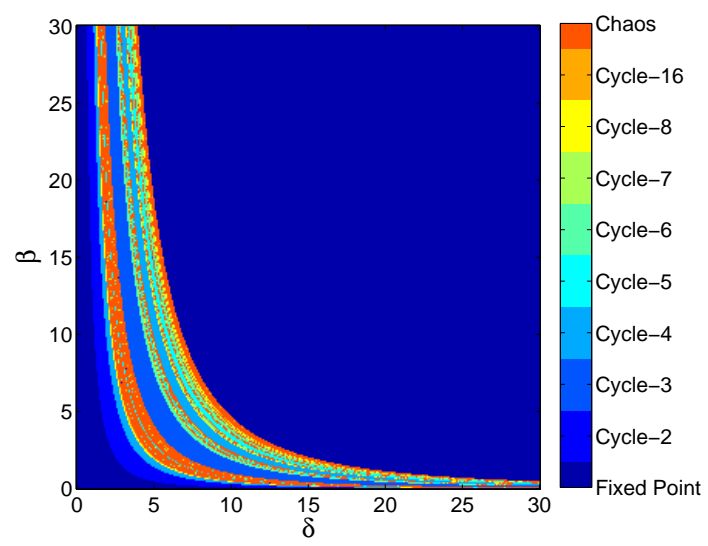

Figure 4: Cycle cartogram in $(\delta, \beta)$ plane for $k=0.1, R=1.05$ and i.c. $x_{0}=x_{m}$.

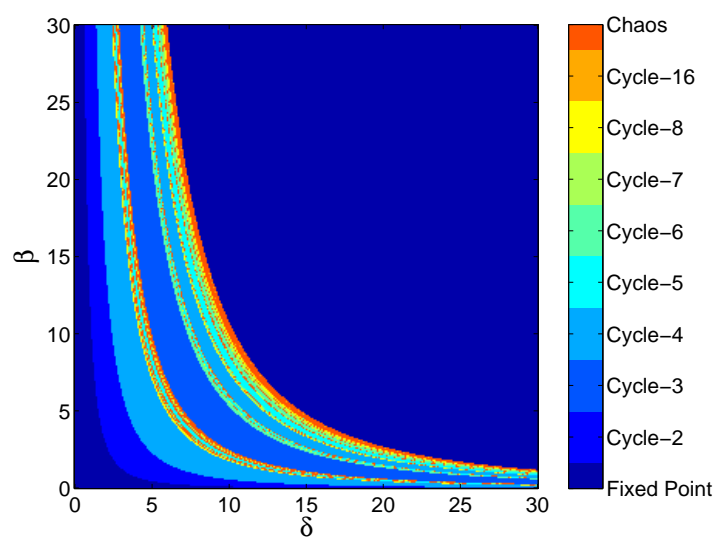

Figure 5: Cycle cartogram in $(\delta, \beta)$ plane for $k=0.07, R=1.05$ and i.c. $x_{0}=x_{m}$.

In figure 4 we present the bifurcation diagram with respect to $\delta$ and $\beta$ with $k=0.1$. Numerical simulations show that the stability region decreases considering decreasing values of $k$. Figure 5 confirms this reasoning: the two dimensional bifurcation diagram has been depicted for $k=0.07$.

Notice that two curves exist in the parameter plane $(\delta, \beta)$, namely $\mathcal{C}_{1}$ and $\mathcal{C}_{2}$, such that the stability region is the union of two sets: the set of pairs $(\delta, \beta)$ below the curve $\mathcal{C}_{1}$ and that of points above $\mathcal{C}_{2}$. For couple of parameters between these two curves, the attractor $\mathcal{A}$ of the map has complicated features.

As we stressed, if $k<k_{1}$ and map $\phi$ has a unique steady state $x_{1}^{\star}$, every initial condition generates 
bounded sequences converging to the unique attractor $\mathcal{A}$, that may consist of the fixed point $x_{1}^{\star}$ or a more complex attractor (periodic or chaotic) located around $x_{1}^{\star}{ }^{2}$ However, according to Lemma 3 , as some parameters vary, the map undergoes a fold bifurcation and two positive new steady states are created, $0<x_{2}^{\star}<x_{3}^{\star}$, one stable and one unstable. Such cases are characterized by coexisting attractors: the attractor $\mathcal{A}$ and the stable steady state $x_{3}^{\star}$. As a consequence, the structure of the boundaries that separate basins of attraction of coexisting attractors becomes prominent. In fact, nonconnected basins of attraction may arise, due to the contact between critical points and basins' boundaries. $^{3}$

In order to investigate the occurrence of contact bifurcations, i.e. global bifurcations responsible for the creation of basins with a fractal boundary, let us focus on the case $k<k_{1}$ with three fixed points, $x_{1}^{\star}<0$ and $0<x_{2}^{\star}<x_{3}^{\star}<\frac{\delta k}{R}$.

For certain parameter values, the graphical analysis of the map $\phi(x)$ shows that the basin of the attractor $\mathcal{A}$ is simply connected and bounded by the unstable fixed point $x_{2}^{\star}$ and its rank-1 preimage $\left(x_{2}^{\star}\right)_{-1}=\phi^{-1}\left(x_{2}^{\star}\right)$, i.e. $B(\mathcal{A})=\left(\left(x_{2}^{\star}\right)_{-1}, x_{2}^{\star}\right)$. Initial conditions taken in the complementary of $B(\mathcal{A})$ generate trajectories converging to the stable steady state $x_{3}^{\star}$, so that the basin of $x_{3}^{\star}$ is formed by the union of two nonconnected portions: $B\left(x_{3}^{\star}\right)=\left(-\infty,\left(x_{2}^{\star}\right)_{-1}\right) \cup\left(x_{2}^{\star},+\infty\right)$, where $B_{1}=\left(x_{2}^{\star},+\infty\right)$ is the immediate basin and $B_{2}=\left(-\infty,\left(x_{2}^{\star}\right)_{-1}\right)=\phi\left(B_{1}\right)$ (see figure 6 panel $(a)$ ).

(a)

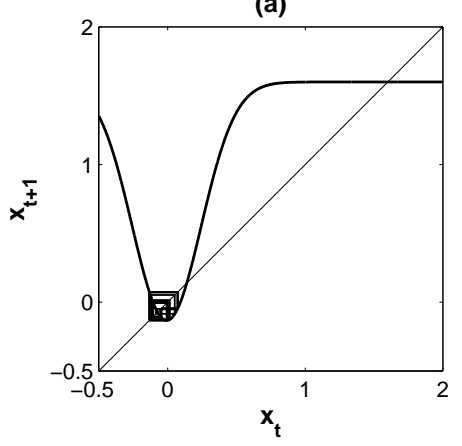

(b)

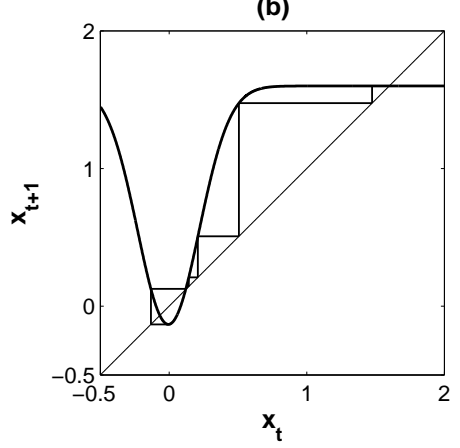

Figure 6: Basins of the attractor $\mathcal{A}$ for $k=0.28<k_{1}, R=1.05$ and $\delta=6$. (a) For $\beta=8$, the trajectory starting from the i.c. $x_{0}=x_{m}$ converges to $\mathcal{A}$. (b) For $\beta=10$, the trajectory starting from the i.c. $x_{0}=x_{m}$ converges to $x_{3}^{\star}$. A contact bifurcation occurred.

The situation drastically changes if the minimum value $\phi\left(x_{m}\right)$ decreases until it crosses the basin boundary $\left(x_{2}^{\star}\right)_{-1}$. In fact at $\phi\left(x_{m}\right)=\left(x_{2}^{\star}\right)_{-1}$ a contact between the critical point and the

\footnotetext{
${ }^{2}$ See Devaney (1989).

${ }^{3}$ See Mira et al. (1996) and Abraham et al. (1997) for an extensive survive of the properties of noninvertible maps.
} 
basin boundary occurs, producing a global bifurcation which changes the structure of the basin. After this bifurcation, new preimages appear and they constitute an infinite and countable set of nonconnected portions and the generic trajectory converges to the stable steady state $x_{3}^{\star}$ (see figure 6 panel $(b)$ ). Notice that the complementary of the union of all these preimages is a Cantor set (with zero Lebesgue measure). As is well-known, the dynamics on the invariant Cantor set can be described by symbolic dynamics. More specifically, the dynamics of $\phi$ on the invariant Cantor set is equivalent to the dynamics of the chaotic shift map on the set of all one-sided symbolic sequences of 0 's and 1's. This means that the dynamics of $\phi$ on the Cantor set is also chaotic.

The presence of nonconneted basins of attraction and the modification in their structure due to the occurrence of contact bifurcations has great importance form the conomic point of view. In terms of policy implications in fact the initial state of the system plays an important role on the final dynamics since two close initial conditions may produce trajectories converging to different attractors.

\section{Returning to the randomness}

This section is devoted to the analysis of some remarkable properties of the stochastic dynamical system. We move from the results reached in the deterministic study of the model. As we did in previous section, we fix the value of $R=1.05$.

\subsection{The measure of the stability region}

???paragrafo riscritto da roy??? Lemma [XXXXX 3 XXXXXX] states the existence of a critical threshold $k_{1}$ such that, for $k \geq k_{1}$ complex dynamics are not allowed. Therefore, it makes sense to provide an estimate of the stability region for $k<k_{1}$. Indeed, if the couple $(\beta, \delta)$ do not belong to the stability region, then map [XXXXX 16 XXXXXX] can exhibit complex dynamics.

We intend here to provide an explicit formulation of an estimate of the measure of the stability region. This analysis allows us to create a set of long-run strategies for each agent taking position in the market. In fact:

- the stability region is related to a kind of "complete information" about the future, due to the nature of the dynamics exhibited by the map;

- the distributions of the stochastic parameters $\beta$ and $\gamma$ coincide with the asymptotic distributions of the stochastic processes $\beta_{t}$ and $\gamma_{t}$. Therefore, the long-run performances are driven by an initial choice.

For each $k<k_{1}$, the stability region is delimited by two bifurcation curves. We denote the lower curve as $\mathcal{C}_{1}^{(k)}$ and the upper as $\mathcal{C}_{2}^{(k)}$. 
We construct two suitable functions describing $\mathcal{C}_{1}^{(k)}$ and $\mathcal{C}_{2}^{(k)}$ by interpolating. The procedure works as follows.

- We consider $k=0.02: 0.0015: 0.29$.

- Fixed $k$, we extract numerically a large enough number ${ }^{4}$ of couples belonging to $\mathcal{C}_{1}^{(k)}$ and $\mathcal{C}_{2}^{(k)}$. We denote them as $\left\{\left(\beta_{1, j}^{(k)}, \delta_{1, j}^{(k)}\right)\right\}_{j=1, \ldots, N_{1}^{(k)}}$ and $\left\{\left(\beta_{2, j}^{(k)}, \delta_{2, j}^{(k)}\right)\right\}_{j=1, \ldots, N_{2}^{(k)}}$, respectively.

- By interpolating 5 , we search for two functions $f_{1}^{(k)}$ and $f_{2}^{(k)}$ providing, respectively, a good approximation of $\mathcal{C}_{1}^{(k)}$ and $\mathcal{C}_{2}^{(k)}$.

- We check the robustness of the best fit procedure.

At the end of the algorithm described above, we are authorized to define the stability region as

$$
\Gamma^{(k)}:=\left\{(\delta, \beta) \in(0,+\infty)^{2} \mid \beta<f_{1}^{(k)}(\delta) \vee \beta>f_{2}^{(k)}(\delta)\right\}, \quad \forall k
$$

Among several available choices, we prefer a power law fit. For each value of $k$, a simple minimum least square procedure gives the existence of four constants $c_{1}^{(k)}, c_{2}^{(k)}, d_{1}^{(k)}, d_{2}^{(k)}, e_{1}^{(k)}$ and $e_{2}^{(k)}$ such that

$$
f_{1}^{(k)} \sim c_{1}^{(k)} \delta^{d_{1}^{(k)}}+e_{1}^{(k)} ; \quad f_{2}^{(k)} \sim c_{2}^{(k)} \delta^{d_{2}^{(k)}}+e_{2}^{(k)}
$$

To test the robustness of the best fit procedure, we compute the R-square parameter between $\left\{c_{i}^{(k)} \delta_{i, j}^{d_{i}^{(k)}}+e_{i}^{(k)}\right\}_{j=1, \ldots, N_{i}^{(k)}}$ and $\left\{\beta_{i, j}\right\}_{j=1, \ldots, N_{i}^{(k)}}$, with $i=1,2$. Fixed $k$, we denote the R-square parameters as $R_{i}^{(k)}$, with $i=1,2$. In Table 1 , the results of our analysis are reported. Their goodness assure the robustness of the best fit procedure.

Table 1

\begin{tabular}{|c|c|c|c|c|}
\hline \hline & Maximum & Minimum & Mean & Standard Deviation \\
\hline \hline$R_{1}$ & 0.9996 & 0.9684 & 0.9856 & 0.0082 \\
\hline$R_{2}$ & 0.9996 & 0.9882 & 0.9977 & 0.0023 \\
\hline
\end{tabular}

We now provide a clarifying example.

Example 5. Assume that $k=0.07$. The best fit procedure is robust (for a visual inspection, see

Figure 7). In this case we obtain:

$$
\begin{gathered}
f_{1}^{(0.07)}(\delta)=13.61 \delta^{-2.135}+0.1082 \\
f_{2}^{(0.07)}(\delta)=909.6 \delta^{-1.889}-0.475
\end{gathered}
$$

\footnotetext{
${ }^{4}$ The number of extracted points vary with $k$, and we denote it with $N_{1}^{(k)}$ for $\mathcal{C}_{1}^{(k)}$ and $N_{2}^{(k)}$ for $\mathcal{C}_{2}^{(k)}$. In our application we consider $N_{1}^{(k)}$ and $N_{2}^{(k)}$ greater than 108, for each $k$.

${ }^{5}$ Matlab ${ }^{\circledR}$ best fit tool has been used.
} 
Therefore, we have:

$$
\Gamma^{(0.07)}=\left\{(\delta, \beta) \in(0,+\infty)^{2} \mid \beta<13.61 \delta^{-2.135}+0.1082 \vee \beta>909.6 \delta^{-1.889}-0.475\right\} .
$$

The probability measure of $\Gamma$ is obtained by using standard stochastic calculus results. We have

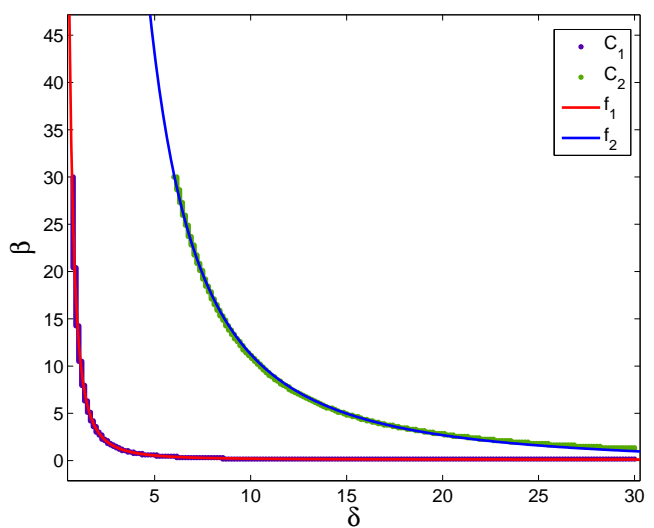

Figure 7: Best fit of the empirical bifurcation curves $\mathcal{C}_{1}^{(k)}$ and $\mathcal{C}_{2}^{(k)}$ for $k=0.07$.

$$
\begin{gathered}
P(\Gamma)=P\left(\{\delta>0\} \cap\left[\left\{0<\beta<13.61 \delta^{-2.135}+0.1082\right\} \cup\left\{\beta>909.6 \delta^{-1.889}-0.475\right\}\right]\right)= \\
=\int_{0}^{13.61 y^{-2.135}+0.1082} f_{\beta \mid \delta}(x \mid y) \mathrm{d} x+\int_{909.6 y^{-1.889}-0.475}^{+\infty} f_{\beta \mid \delta}(x \mid y) \mathrm{d} x,
\end{gathered}
$$

where $f_{\beta \mid \delta}$ is the conditional density function of $\beta$ given $\delta$. By the knowledge of $f_{\beta \mid \delta}$, we obtain an estimate of the probability of $\Gamma$.

The result previously obtain enable us to know the probability that, for a given value of the confidence parameter, the system will converge to a globally stable steady state. More precisely, when $k$ is small enough and complicated dynamics can be exhibited, once known the probability distributions of $\delta$ and $\beta$, it is possible to know the probability that prices, in the long run, will converge to their equilibrium value. Such a point is characterized by the equlibrium price belove its fundamental value and the market of both groups of agents. Furthermore it is easy to verify that the measure of the stability region depends on the shape of the probability distribution of $\delta$. In particular, a simple analysis of the fit function shows that the measure of $\Gamma$ grows as the distribution of $\delta$ is concentrated in small values.

\subsection{A bayesian analysis: distribution of $\beta$}

???paragrafo non toccato: roy ragionare su dove mettere, possibile estensione e commenti economici??? In this subsection we propose a method for choosing the belief rule by performing forecasts on the fundamental value. To this end, we analyze the model from a bayesian point of view and we do not explicate the values of $k$. 
Our purpose is to provide a long-run equilibrium value for the fundamental, by estimating the evolution of $\delta_{t}$ and setting a limit as $t$ goes to infinity, and then describing the factor $\beta_{t}$, that gives information on the heterogeneity and the explicit form of the agents' beliefs on the future prices.

The bifurcation curves are well approximated by using a sum of gaussian functions, as remarked in the previous subsection. Hence, from the knowledge of the value of the parameter $\delta$, we derive the distribution of the parameter $\beta$. We have an explicit approximate formula for the bounds of the region $\Gamma$ as follows:

$$
\begin{aligned}
& f_{1}(\delta)=\sum_{k=1}^{+\infty} a_{1, k} \exp \left[\left(-\left(\delta-b_{1, k}\right) / c_{1, k}\right)^{2}\right] \\
& f_{2}(\delta)=\sum_{k=1}^{+\infty} a_{2, k} \exp \left[\left(-\left(\delta-b_{2, k}\right) / c_{2, k}\right)^{2}\right]
\end{aligned}
$$

where $a_{j, k}, b_{j, k}$ and $c_{j, k}$ are opportunely chosen real numbers. Let us define the curves

$$
\begin{aligned}
& \mathcal{C}_{1}=\left\{\beta=f_{1}(\delta), \delta>0\right\}, \\
& \mathcal{C}_{2}=\left\{\beta=f_{2}(\delta), \delta>0\right\} .
\end{aligned}
$$

If the value of $\delta$ is known, then $\beta$ faces a probability density function that is a mixture of infinite gaussians, in both of the cases $\mathcal{C}_{1}$ and $\mathcal{C}_{2}$.

For each $t \in \mathbb{N}$, a couple of bifurcation curves $\mathcal{C}_{1, t}$ and $\mathcal{C}_{2, t}$ exist converging to the asymptotic curves defined in (21) and (22), as $t \rightarrow+\infty$. The following proposition states immediately:

Proposition 6. Fix $t \in \mathbb{N}$. Let us assume that $\mathcal{C}_{1, t}$ and $\mathcal{C}_{2, t}$ are defined, respectively, by the functions $f_{1, t}$ and $f_{2, t}$, where

$$
\begin{aligned}
& f_{1, t}=\sum_{k=1}^{t} a_{1, k} \exp \left[\left(-\left(\delta-b_{1, k}\right) / c_{1, k}\right)^{2}\right], \\
& f_{2, t}=\sum_{k=1}^{t} a_{2, k} \exp \left[\left(-\left(\delta-b_{2, k}\right) / c_{2, k}\right)^{2}\right] .
\end{aligned}
$$

Then

$$
\lim _{t \rightarrow+\infty} f_{j, t}=f_{j}, \quad j=1,2 .
$$

According to Proposition 6, if an agent in the market makes forecasts at time $t$ on the long-run fundamental value $\delta$, then she can calibrate her own adjustment parameter $\beta_{t}$ by using a mixture of $t$ gaussians.

Furthermore, since $\beta_{t}$ is a mixture of gaussian distributions, then it can be written by an autoregressive simple rule:

$$
\beta_{t}=\beta_{t-1}+\epsilon_{t}
$$

with $\epsilon_{t} \sim N\left(\mu_{\epsilon}(t), \sigma_{\epsilon}^{2}(t)\right)$ and $\epsilon_{t_{1}}$ independent of $\epsilon_{t_{2}}$, for each $t_{1} \neq t_{2}, t_{1}, t_{2} \in \mathbb{N}$. Moreover, the stability property of the gaussian distribution and the independence of $\epsilon$ 's imply that $\beta_{t} \sim$ 
$N\left(\mu_{\beta}(t), \sigma_{\beta}^{2}(t)\right)$, with

$$
\mu_{\beta}(t)=\sum_{k=1}^{t} \mu_{\epsilon}(k), \quad \sigma_{\beta}^{2}(t)=\sum_{k=1}^{t} \sigma_{\epsilon}^{2}(k) .
$$

The adjustment parameter at time $t$ can be obtained by the adjustment parameter at time $t-1$ added with a gaussian error, not necessarily with zero mean. Then, the proportion factor $\beta_{t}$ satisfied a learning rule based on the previous values of this parameter. Such a rule is biased by a stochastic noise.

\subsection{A bayesian analysis: the changing in persistence of the beliefs}

???che fare di questo paragrafo: il referee dice che va approfondito ed inserite interpretazioni economiche...??? In this subsection we explore the possible presence of a change in persistence of the investor's beliefs, on the boundary of the stability region. The change in persistence is a statistical concept related to the coexistence in the same time series of two different behaviors: stationarity with respect to a trend (in this case we have an integrated process of order $0, \mathrm{I}(0)$ ); presence of a stochastic trend (in this case we speak about an integrated process of order 1, I(1)). If a change in persistence occurs in a sample period $\{1, \ldots, t\}$, then a breakpoint $\tau \in(0,1)$ exists such that the time series $\beta_{k}$ is $\mathrm{I}(0)$ (or $\left.\mathrm{I}(1)\right)$, for $k=1, \ldots,[\tau t]$, and it is $\mathrm{I}(1)$ (or $\left.\mathrm{I}(0)\right)$ for $k=[\tau t]+1, \ldots, t$, where $[\tau t]$ indicates the inferior integer part of $\tau t$. In our stochastic framework, this means that the change in fundamentalist-chartist proportion is ruled by a very different perception of the deviation from the fundamental: in the stationary case, the beliefs at different times show a common deterministic trend, and do not differ from each other; in the nonstationary case, the beliefs are driven by an increasing (or decreasing) parameter, and this implies a growing confidence in the deviation from the fundamental. Since we focus on the bifurcation curves $\mathcal{C}_{1, t}$ and $\mathcal{C}_{2, t}$, the switch due to a change in persistence is able to provide information about the stability region, and so about the steady states of the dynamical systems proposed in our framework.

Consider now a fixed value of $\delta$. Then the corresponding $\beta$ is a real number. Hence, we obtain that $\beta_{t}$ shows a constant asymptotic behavior, as $t \rightarrow+\infty$. Statistically, this means that $\beta_{t}$ is a stationary process at least in the last subsample of the sample period. If a change in persistence occurs, it is in the direction from $\mathrm{I}(1)$ to $\mathrm{I}(0)$ (figure 8 shows an example of the evolution of $\beta_{t}$ in a sample period, fixing the value of $\delta$ ).

To explore this phenomenon, we construct a model showing the case of change in persistence in the direction from $\mathrm{I}(1)$ to $\mathrm{I}(0)$. We rewrite $\beta_{k}$ in line with (23) as follows:

$$
\beta_{k}=\rho_{k}+\xi_{k}, \quad k=1, \ldots, t
$$

where $\xi_{k}$ and $\rho_{k}$ are mutually independent i.i.d. gaussian processes with same mean $\mu$ and variance 


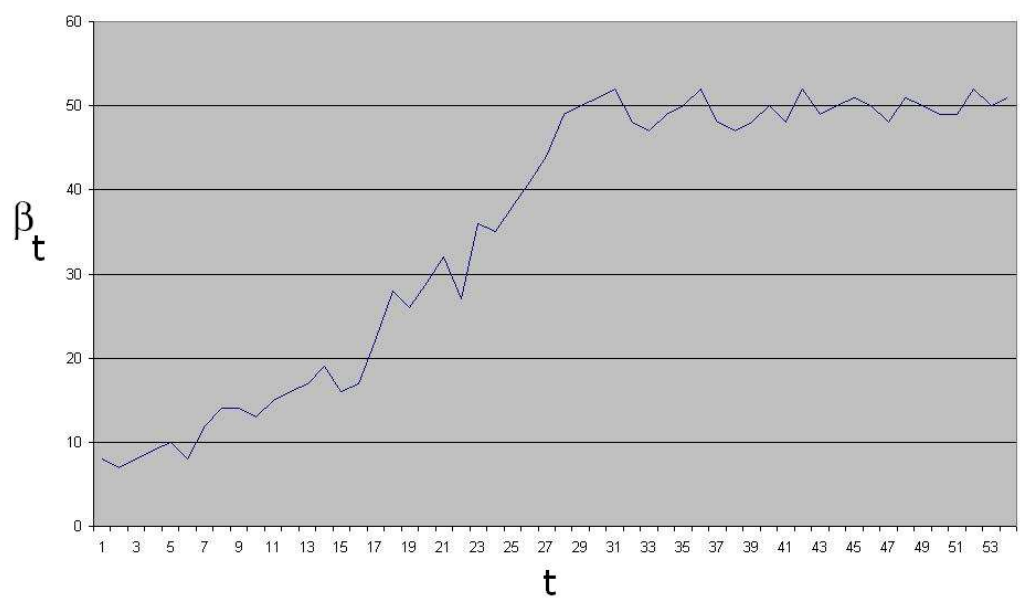

Figure 8: Evolution of $\beta_{t}$ in a sample period $\{1, \ldots 53\}$, fixing the value of $\delta$.

$\sigma^{2}$, with

$$
\mu=\frac{\mu_{\beta}(t)}{2 t-1}, \quad \sigma^{2}=\frac{\sigma_{\beta}^{2}(t)}{2 t-1} .
$$

Now we introduce the presence of a stochastic error in the dynamic described by (24). We assume that $\rho$ follows an evolution rule able to explore the eventual presence of a change in persistence in the dynamic of $\beta$. We have

$$
\rho_{k}=\rho_{k-1}+1(k \leq[\tau t]) \eta_{k}, \quad k=1, \ldots t
$$

where $1(\cdot)$ is the indicator function, and $\eta_{k}$ is an i.i.d. gaussian stochastic process that is mutually independent of $\xi_{k}$ with mean zero and variance $\sigma_{\eta}^{2}$. The time-dependent parameter $\beta_{k}$ yields a process which is nonstationary up to and including time $[\tau t]$ but it is $\mathrm{I}(0)$ after the break, if and only if $\sigma_{\eta}^{2}>0$. In the converse case, we have that $\sigma_{\eta}^{2}=0$, and the process is stationary in the whole sample period. In this case, a change in persistence does not occur. Formally, the statistical tests devoted to estimating the presence of change in persistence have null hypothesis $H_{0}: \sigma_{\eta}^{2}=0$ and alternative hypothesis $H_{1}: \sigma_{\eta}^{2}>0$. We suggest the statistics proposed by Kim (2000), Busetti and Taylor (2004) in order to explore the presence of a change in persistence.

\subsection{A bayesian analysis: theoretical cluster analysis of the data}

???che fare di questo paragrafo: il referee dice che va approfondito ed inserite interpretazioni economiche...??? The basic idea of this last remark is to derive a theoretical methodology able to classify the parameter $\beta_{t}$ as the value of $\delta$ changes. We aim to propose a clustering of $\beta_{t}$ with respect to their ranges of variation, in the boundary of the stability region $\Gamma$. This analysis allows us to obtain information about the bifurcations of the dynamical system, in agreement with the deterministic study. 
Let us fix $\delta=\bar{\delta}$, with $\bar{\delta} \in D$, where $D$ is defined in subsection 4.1. For each $\bar{t} \in \mathbb{N}$, the fact that $\beta_{\bar{t}}$ is a gaussian distribution implies that it is uniquely determined by its mean and variance. We construct a 3-dimensional grid

$$
G:=\left\{\left(\delta_{k}, \mu_{k, t}, \sigma_{k, t}^{2}\right) \in D \times(0,+\infty)^{2} \quad \forall t, k \in \mathbb{N}\right\}
$$

where $\mu_{k, t}$ and $\sigma_{k, t}^{2}$ are, respectively, the mean and the variance of $\beta_{t}$ conditioned to $\delta=\delta_{k}$.

The mixture of gaussians model is a powerful estimation of the parameters $\mu$ 's and $\sigma^{2}$ 's, and so of the distribution of the $\beta$ 's. The model assumes that the data are produced by a mixture of $\mathrm{N}$ multivariate gaussians.

The cluster classes we consider are related to the joint mean variance size of the $\beta$ 's, and they are obtained by considering a suitable partition of $(0,+\infty)^{2}$. In this way, we have information about how investors calibrate their beliefs by performing a forecast on the fundamental. The mean of the $\beta$ 's represents the degree of confidence on the fundamentals expected value (the biggest is the mean of $\beta_{t}$, the highest is the distortion of the fundamentals expected value at time $t+1$, given the information available at time $t$ ); the variance of the $\beta$ 's measures the stability of the distortion, and represents an error that the investor makes about the degree of confidence in the fundamental expected value. Such an error is proportional to the variance's size.

In our framework, the plots of the grid $G$ are obtained by a mixture of gaussians. We suggest a couple of cluster procedures, involving mixture of gaussians data.

The first one is the standard clustering EM procedure (see Dempster et al., 1977) allowing to distinguish some classes within the grid $G$.

A more sophisticated cluster techniques is the Kohonen algorithm, based on the Voronoi tessellation of the grid $G$ and on the introduction of some cluster weights. For details of the Kohonen network, we remind the reader of Kohonen (1989, 1991a, 1991b). A sufficient condition for the convergence of the algorithm in the multidimensional case can be found in Feng and Tirozzi (1997), Lin and Si (1998) and Sadeghi (2001).

\section{Conclusions}

????rivedere inserendo commenti economici: ci pensa eli??? This paper deals with an stochastic agent-based asset pricing model. In a contest with bounded rationality we assume that agents form their expectations on future prices not only on the basis of past observations but also by taking into account the type-distribution of agents. In this new contest we introduce a sort of confidence adjustment coefficient on the updating rule, hence we rely on a market populated by fundamentalists and chartists following our new approach. The dynamical map is bidimensional and it exhibits randomness. We studied it by proving results related to the deterministic skeleton 
of the system and we proved that when most agents are chartists (fundamentalists) in type and the forecast of the fundamental provides small (high) values, only simple dynamics is possible and there is a stability region in the parameters' plane for which the only possible dynamics is convergence to a steady state. The system shows complex dynamics in the remaining cases.

The stability region was also analyzed by reintroducing stochasticity and we provided an explicit formula for its probability measure. Finally, we proposed a bayesian analysis, in order to explore the distribution of the adjustment term of the proportion of agents.

\section{References}

[1] Abraham, R., Gardini, L., Mira, C., 1997. Chaos in discrete dynamical systems (a visual introduction in two dimension), Springer-Verlag.

[2] Avery, C., Zemsky, P., 1998. Multidimensional Uncertainty and Herd Behavior in Financial Markets. American Economic Review, 88, (4), 724-748.

[3] Banerjee, A., 1992. A simple model of herd behavior. Quarterly Journal of Economics, 107, 787-818.

[4] Bikhchandani, S., Hirshleifer, D., Welch, I., 1992. A theory of fads, fashion, custom and cultural change as informational cascades. Journal of Political Economy, 100, 992-1027.

[5] Brock, W.A., Hommes, C.H., 1997. Rational route to randomness. Econometrica, 65, 1059-1095.

[6] Brock, W. A., Hommes, C. H., 1998. Heterogeneous beliefs and routes to chaos in a simple asset pricing model. Journal of economic dynamics and control, 22, 1235-1274.

[7] Busetti, F. and A.M.R., Taylor, 2004. Tests of stationarity against a change in persistence. Journal of Econometrics 123, 33-66.

[8] Chamley, C., Gale, D., 1994. Information revelation and strategic delay in a model of investment. Econometrica, 62, 1065-1086.

[9] Chari, V., Kehoe, P., 2000. Financial crises as herds. Federal Reserve Bank of Minneapolis, Working Paper 600.

[10] Conlisk, J., 1996. Why bounded rationality? Journal of Economic Literature, 34, 669-700.

[11] Dempster A.P., Laird N.M., and Rubin D.B., 1977. Maximum Likelihood from Incomplete Data via the EM algorithm Journal of the Royal Statistical Society, Series B, vol. 39, 1:1-38

[12] Devaney, R.L., 1989. An introduction to chaotic dynamical system. The Benjamin/Cummings Publishing Co, Menlo Park. 
[13] Feng, J.F., Tirozzi B., 1997. Convergence Theorem for the Kohonen Feature Mapping Algorithms with VLRPs, Computer Math. Applic., 33, 3, 45-63.

[14] Hommes, C. H., 1994. Dynamics of the cobweb model with adaptive expectations and nonlinear supply and demand. Journal of Economic Behavior and Organization, 24, 315-335.

[15] Hommes, C. H., 2001. Financial Markets as nonlinear adaptive evolutionary systems. Quantitative Finance, 1, 149-167.

[16] Hommes, C. H., 2006. Heterogeneous agent models in economics and finance. In Handbook of Computational Economics, L. Tesfatsion and K. L. Judd, eds., vol. 2, chap. 23. Elsevier, North Holland, 1109-1186.

[17] Kim, J.Y., 2000. Detection of change in persistence of a linear time series. Journal of Econometrics 95, 97-116.

[18] Kirman, A.P., 2006. Heterogeneity in economics. Journal of Economic Interaction and Coordination, 1, 89-117.

[19] Kohonen, T., 1989. Self-Organization and Associative Memory Process, (Springer-Verlag).

[20] Kohonen, T., 1991a. Analysis of a Simple Self-Organizing Process, Biological Cybernetics, 44, $135-140$.

[21] Kohonen, T., 1991b. Self-Organizing maps: optimization approaches, Artificial Neural Networks, 1, 981-990.

[22] Lin, S., Si, J., 1998. Weight-Value Convergence of the SOM Algorithm for Discrete Input, Neural Computation, 10, 807-814.

[23] Mira, C., Gardini, L., Barugola, A., Cathala, J. C., 1996. Chaotic dynamics in two-dimensional noninvertible maps. World Scientific, Singapore.

[24] Sadeghi, A.A., 2001. Convergence in Distribution of the Multidimensional Kohonen Algorithm, Journal of Applied Probability, 38, 136-151.

[25] Simon, H., 1957. Models of man. John Wiley \& Sons, New York. 